\title{
SUBNORMALITY CONDITIONS IN NON-TORSION GROUPS
}

\author{
Luise-Charlotte Kappe and Gunnar Traustason
}

According to results of Heineken and Stadelmann, a non-torsion group is a 2-Baer group if and only if it is 2-Engel, and it has all subgroups 2-subnormal if and only if it is nilpotent of class 2. We extend some of these results to values of $n$ greater than 2 . Any non-torsion group which is an $n$-Baer group is an $n$-Engel group. The converse holds for $n=3$, and for all $n$ in the case of metabelian groups. A non-torsion group without involutions having all subgroups 3 -subnormal has nilpotency class 4 , and this bound is sharp.

\section{INTRODUCTION}

Let $G$ be a group. A subgroup $H$ in $G$ is said to be subnormal, if there exists a finite series $H=H_{0}, H_{1}, \ldots, H_{k-1}, H_{k}=G$, such that

$$
H=H_{0} \triangleleft H_{1} \triangleleft \cdots \triangleleft H_{k}=G .
$$

If $n$ is the length of the shortest such series, we say $H$ is subnormal of defect $n$, or $n$ subnormal, denoted by $H \triangleleft_{n} G$. Now let $H$ be an arbitrary subgroup of $G$. We define the series, $\left(H^{G, i}\right)_{i=1}^{\infty}$, of successive normal closures by induction as follows:

$$
H^{(G, 0)}=G, \quad H^{(G, i+1)}=H^{H^{(G, i)}}
$$

where $H^{K}$ denotes as usual the normal closure of $H$ in $K$. It is easy to see that $H$ is subnormal in $G$ if and only if $H^{(G, r)}=H$ for some $r$, and the smallest integer $n$ such that $H^{(G, n)}=H$ is the subnormal defect of $H$ in $G$.

In a group of nilpotency class $n$, all subgroups are subnormal of defect at most $n$. Conversely, Roseblade in [8] has shown that a group $G$ with all subgroups $n$-subnormal is nilpotent of class $\mu(n)$. However, $\mu(n)$ is not explicitly given in [8] and the exact values are only known for $n=1$ and 2 . So far, the function $\mu(n)$ seems to be not well understood. On the one hand, using direct methods following Roseblade's approach, it appears that $\mu(n)$ grows very rapidly with $n$. On the other hand, it can be easily seen that in metabelian groups $\mu(n)$ grows linearly with $n$, provided the group does not contain elements of small order (see for example [3]).

Received 9th November, 1998

Copyright Clearance Centre, Inc. Serial-fee code: 0004-9729/99 \$A2.00+0.00. 
Set $[x, k y]=[[x, k-1, y], y]$ and $[x, 1 y]=[x, y]=x^{-1} y^{-1} x y$. An element $x$ in a group $G$ is a right $n$-Engel element in $G$ if $[x, n y]=1$ and a left $n$-Engel element if $\left[y,_{n} x\right]=1$ for all $y$ in $G$. A group is called an $n$-Engel group if every element in the group is left and right $n$-Engel.

A group with all cyclic subgroups $n$-subnormal is called an $n$-Baer group. The class of $n$-Baer groups will be denoted by $B_{n}$. It can be easily seen that any group in $B_{n}$ is $(n+1)$-Engel. Denoting the class of groups with all subgroups $n$-subnormal by $U_{n}$, we obviously have that every $U_{n}$-group is a $B_{n}$-group. In case $n=1$, the class of Dedekind groups (see $[1,2]$ or $[9$, Theorem 6.1.1] for easier reference), all subgroups being $n$ subnormal is equivalent to all cyclic subgroups being $n$-subnormal. This is no longer the case if $n \geqslant 2$. For instance, $n$-Baer groups, $n \geqslant 3$, are not necessarily nilpotent. However, 2-Baer groups are nilpotent. In fact, results of Heineken [5] and Mahdavianary [7] state that a group with all cyclic subgroups 2-subnormal is nilpotent of class not exceeding 3 . As a corollary of this result, it follows that $\mu(2) \leqslant 3$. This bound is sharp. However not all $B_{2}$-groups are $U_{2}$-groups.

In this article we are interested in the non-torsion groups in $U_{n}$ and $B_{n}$. There is some evidence that the structure of the non-torsion groups in these classes is different. A non-torsion group is for example a Dedekind group if and only if it is Abelian. Heineken [5] has also shown that a non-torsion group is a 2-Baer group if and only if it is 2-Engel, whereas a result of Stadelmann [10] states that a non-torsion group is a $U_{2}$-group if and only if it is nilpotent of class at most 2. Furthermore, in [3] it was shown that for metabelian non-torsion groups, $n$-Engel and $n$-Baer are equivalent, provided $n \leqslant 5$, or the group contains no elements of order $\leqslant n-1$ in case $n \geqslant 6$. In this article we shall show that this result holds without restrictions on element orders.

It is natural to ask whether one can extend these results. We shall see that one implication in Heineken's result can be generalised. We shall show that every non-torsion $n$-Baer group is always an $n$-Engel group, and in fact, we have that these conditions are equivalent when $n=3$. One expects that in $U_{n}$, the non-torsion groups have a much simpler structure than the torsion groups. At this time we do not have an analogue of Stadelmann's Theorem for general $n$. However, we can say a few things about $U_{3}$-groups. In $[11]$ it is shown that every torsion-free $U_{3}$-group is nilpotent of class not exceeding 3 . However, this result does not extend to non-torsion groups in $U_{3}$ in general, as we shall see in this paper. It follows from another result in [11] that non-torsion groups in $U_{3}$ without involution have nilpotency class at most 4 . This bound is sharp since, as we see from Theorem 3.1, there exists a family of non-torsion groups in $U_{3}$ with nilpotency class 4 and with torsion subgroup of $p$-power order, $p \neq 2$. For non-torsion groups in $U_{3}$ with involutions, as we see in Proposition 3.4, the class bound is at least 4 . We do not know at present if this is an upper bound on the nilpotency class. 


\section{NON-TORSION GROUPS WITH EVERY CYCLIC SUBGROUP $n$-SUBNORMAL}

In this section we prove our first main result, that every non-torsion $n$-Baer group is an $n$-Engel group, extending Heineken's result for $n=2$ [5]. We begin with an elementary lemma for nilpotent groups.

LEMma 2.1. Let $G$ be a nilpotent group of class $c$ in which the torsion subgroup $\tau(G)$ has finite exponent $r$. Then $\left[\tau(G), G^{r^{c-1}}\right]=1$.

Proof: We show by induction that

$$
\left[\tau(G)_{c-i} G, G^{r^{i-1}}\right]=1
$$

for $i=1,2, \ldots, c$. Since $G$ is nilpotent of class $c$ this is clearly true for $i=1$. For the induction step we assume that this is true for $i=1, \ldots, k$ for some $1 \leqslant k<c$. Let $x \in\left[\tau(G)_{c-(k+1)} G\right]$ and let $g \in G$. From the induction hypothesis we have that

$$
\begin{aligned}
{\left[x, u g^{r^{k-1}}\right] } & =\left[x, g^{r^{k-1}}\right][x, u]\left[x, u, g^{r^{k-1}}\right] \\
& =\left[x, g^{r^{k-1}}\right][x, u]
\end{aligned}
$$

for all $u \in G$. Using this repeatedly we get

$$
\left[x, g^{r^{k}}\right]=\left[x, g^{\left(r^{k-1}\right) r}\right]=\left[x, g^{r^{k-1}}\right]^{r}=1,
$$

since $\tau(G)^{r}=1$. This finishes the proof of the inductive step. In particular we have $\left[\tau(G), G^{r^{c-1}}\right]=1$.

Our aim is to show that every element in a non-torsion $n$-Baer group is a left $n$-Engel element. In our next lemma we show this for the elements of infinite order.

LEMMA 2.2. Let $G$ be an $n$-Baer group. Then every element of infinite order is a left $n$-Engel element.

Proof: Suppose $x, y \in G$ with $|y|=\infty$. Since $G$ is an $n$-Baer group, we have $\langle y\rangle^{(G, n)}=\langle y\rangle$. It follows that $\left[y,\left[x_{n-1} y\right]\right]=y^{r}$ for some $r \in Z$. Since $G \in B_{n}$, we have that $G$ is $(n+1)$-Engel. Hence

$$
1=\left[y_{n+1}\left[x_{, n-1} y\right]\right]=y^{r^{n+1}}
$$

Since $|y|=\infty$, we must have $r=0$.

TheOREM 2.3. Let $G$ be a non-torsion $n$-Baer group. Then $G$ is an $n$-Engel group.

Proof: By the last lemma, every element of infinite order is a left $n$-Engel element. We thus only have to show that every element of finite order is a left $n$-Engel element. We first show that every element of infinite order is a right $n$-Engel element. Let $z, x \in G$ 
such that $|z|=\infty$ and $|x|<\infty$. Then $H=\langle z, x\rangle$ is a finitely generated nilpotent group and thus $\tau(H)$ is finite. By Lemma 2.1 we have that $H / C_{H}(\tau(H))$ is of finite exponent, say $m$. Then, since $\left|x z^{m}\right|=\infty$, we have that

$$
\begin{aligned}
1 & =\left[z,_{n} x z^{m}\right] \\
& =\left[[z, x]\left[z, x, z^{m}\right]_{, n-1} x z^{m}\right] \\
& =\left[z, x,_{n-1} x z^{m}\right] \\
& =\left[z,_{n} x\right] .
\end{aligned}
$$

So we have shown that every element of infinite order is a right $n$-Engel element. In particular, if $|y|,|x|<\infty$ and $|z|=\infty$ then $1=\left[y z^{m}{ }_{n} x\right]$. Since $\langle x, y, z\rangle$ is nilpotent, we can apply Lemma 2.1 again to find another $m$ such that $z^{m}$ commutes with $x$ and $y$. But then

$$
1=\left[y z^{m},_{n} x\right]=\left[y,_{n} x\right]
$$

and we have proved the theorem.

Corollary 2.4. A non-torsion group is a 3-Engel group if and only if it is a 3-Baer group.

ProOF: By Theorem 2.3, a non-torsion group in $B_{3}$ is 3-Engel. Conversely, by [6] we have for any group $G$ the conditions $G$ being 3-Engel and the normal closure $x^{G}$ of every element $x$ in $G$ having nilpotency class 2 are equivalent. Hence $\langle x\rangle \triangleleft_{2} x^{G}$. Since $x^{G} \triangleleft G$, it follows that $\langle x\rangle \triangleleft_{3} G$, the desired result.

The result for $n=2$, corresponding to the above corollary, is due to Heineken [5]. The result of the next corollary appears in [3] for $n \leqslant 5$ and for $n \geqslant 6$, provided the group contains no elements of order $\leqslant n-1$.

Corollary 2.5. A metabelian non-torsion group is an $n$-Engel group if and only if it is an $n$-Baer group.

PROOF: Since $G$ is a non-torsion group in $B_{n}$, it follows by Theorem 2.3 that $G$ is $n$-Engel. By [3, Lemma 2.6] we have that any metabelian $n$-Engel group is an $n$-Baer group, the desired result.

\section{NON-TORSION GROUPS WITH EVERY SUBGROUP 3-SUBNORMAL}

As mentioned in the introduction, Stadelmann [10] has shown that a non-torsion group is a $U_{2}$-group if and only if it is nilpotent of class at most 2 . The topic of this section is the investigation of bounds for the nilpotency class of non-torsion groups in $U_{3}$. A subgroup $H$ of a group $G$ is 3-subnormal in $G$ if and only if $[G, H, H, H] \leqslant H$. Thus any group of class 3 is in $U_{3}$. In [11] it was shown that the converse holds for torsion-free groups. However, as we shall see in the next theorem, this result cannot be extended to non-torsion groups in general. 
THEOREM 3.1. A non-torsion group in $U_{3}$ without involutions has nilpotency class at most four. There exist non-torsion groups without involutions in $U_{3}$ having nilpotency class precisely four.

Proof: Let $G$ be a group as in the hypothesis. Then $G$ is 3-Engel by Theorem 2.3. Since $G$ has no involutions, it follows by [11, Theorem 1] that the nilpotency class of $G$ does not exceed four. Consider the groups of Example 3.2. They have nilpotency class precisely four and are $U_{3}$-groups by Proposition 3.3. Thus the bound on the nilpotency class is sharp.

In the following, we construct a non-torsion group whose torsion subgroup is a $p$ group, $p$ an odd prime, which has the properties claimed in Theorem 3.1 .

EXAMPLE 3.2. Let $p$ be an arbitrary prime different from 2. Take the relatively free group $\langle x, y, z\rangle$ in the variety of 3-Engel groups that are nilpotent of class at most 4 and let $G(p)$ be the quotient satisfying the additional relations:

$$
\begin{array}{llll}
y^{p^{2}}=1, & {[y, z]=y^{2 p},} & {[x, z, z]=1,} & z^{p^{2}}=[y, x, z, x] \\
{[z, x]^{p}=1,} & {[x, y, y]=1,} & {[y, x]^{p}=[y, x, z] .} &
\end{array}
$$

Let $a_{1}=x, a_{2}=z, a_{3}=y, a_{4}=x^{p}, a_{5}=z^{p}, a_{6}=y^{p}, a_{7}=[z, x], a_{8}=[y, x], a_{9}=[y, x]^{p}$, $a_{10}=[z, x, x], a_{11}=[y, x, x]$ and $a_{12}=[y, x, z, x]$. We can deduce from these relations that $G(p) /\left\langle x^{p^{2}}\right\rangle$ has power-commutator presentation with generators $a_{1}, \ldots, a_{12}$ and the following relations:

$$
\begin{aligned}
& a_{1}^{p}=a_{4}, \quad a_{2}^{p}=a_{5}, \quad a_{3}^{p}=a_{6}, \quad a_{4}^{p}=1, \quad a_{5}^{p}=a_{12}, \quad a_{6}^{p}=1, \\
& a_{7}^{p}=1, \quad a_{8}^{p}=a_{9}, \quad a_{9}^{p}=1, \quad a_{10}^{p}=1, \quad a_{11}^{p}=a_{12}, \quad a_{12}^{p}=1, \\
& {\left[a_{2}, a_{1}\right]=a_{7}, \quad\left[a_{3}, a_{1}\right]=a_{8}, \quad\left[a_{3}, a_{2}\right]=a_{6}^{2}, \quad\left[a_{4}, a_{1}\right]=1, \quad\left[a_{4}, a_{2}\right]=1,} \\
& {\left[a_{4}, a_{3}\right]=a_{9}^{p-1} a_{12}^{(p+1) / 2}, \quad\left[a_{5}, a_{i}\right]=1, \quad\left[a_{6}, a_{1}\right]=a_{9}, \quad\left[a_{6}, a_{i}\right]=1 \text { if } i \neq 1,} \\
& {\left[a_{7}, a_{1}\right]=a_{10}, \quad\left[a_{7}, a_{2}\right]=1, \quad\left[a_{7}, a_{3}\right]=a_{9}^{p-1} a_{12}^{4}, \quad\left[a_{7}, a_{i}\right]=1 \text { if } i \geqslant 4,} \\
& {\left[a_{8}, a_{1}\right]=a_{11}, \quad\left[a_{8}, a_{2}\right]=a_{9}, \quad\left[a_{8}, a_{3}\right]=1, \quad\left[a_{8}, a_{4}\right]=a_{12}, \quad\left[a_{8}, a_{5}\right]=1,} \\
& {\left[a_{8}, a_{6}\right]=1, \quad\left[a_{8}, a_{7}\right]=a_{12}^{4}, \quad\left[a_{9}, a_{1}\right]=a_{12}, \quad\left[a_{9}, a_{i}\right]=1 \text { if } i \neq 1,} \\
& {\left[a_{10}, a_{3}\right]=a_{12}^{3}, \quad\left[a_{10}, a_{i}\right]=1 \text { if } i \neq 3, \quad\left[a_{11}, a_{2}\right]=a_{12}^{p-3}, \quad\left[a_{11}, a_{i}\right]=1 \text { if } i \neq 2,} \\
& {\left[a_{12}, a_{i}\right]=1 .}
\end{aligned}
$$

We refer to [12] for a discussion of power-commutator presentations. One can check that this power-commutator presentation is consistent. It follows that $G(p)$ has class 4 .

This concludes the construction of the example. In the next proposition we shall establish that all subgroups of $G(p)$ are 3-subnormal.

PROPOSITION 3.3. For each prime $p \neq 2$ we have that $G(p)$ is in $U_{3}$.

PRoof: Let $g, h_{1}, h_{2}, h_{3} \in G(p)$ and $H=\left\langle h_{1}, h_{2}, h_{3}\right\rangle$. It is sufficient to show that $\left[g, h_{1}, h_{2}, h_{3}\right] \in H$. For ease of notation write $G(p)=G$. We consider several cases. First assume that $H$ contains an element of finite order that is not contained in 
$\langle x, y\rangle[G, G] G^{p}$. Then $H$ has an element of the form $z y^{r} u$, where $r$ is some integer and $u \in\left\langle a_{6}, a_{7}, \ldots, a_{12}\right\rangle$. From the presentation above one sees that $\left\langle a_{6}, a_{9}, a_{12}\right\rangle \triangleleft G$ and that $\left\langle a_{2}, a_{3}, a_{6}, \ldots, a_{12}\right\rangle /\left\langle a_{6}, a_{9}, a_{12}\right\rangle$ is Abelian. Since $y^{p}, u^{p} \in\left\langle a_{6}, a_{9}, a_{12}\right\rangle$, we thus have that

$$
\left(z y^{r} u\right)^{p}=z^{p} v
$$

with $v \in\left\langle a_{6}, a_{9}, a_{12}\right\rangle$. The group $\left\langle a_{5}, a_{6}, a_{9}, a_{12}\right\rangle$ is Abelian and $a_{6}^{p}=a_{9}^{p}=a_{12}^{p}=1$. Therefore

$$
\left(z y^{r} u\right)^{p^{2}}=\left(z^{p} v\right)^{p}=z^{p^{2}}=[y, x, z, x]
$$

and $H$ contains $\gamma_{4}(G)$. In particular $[G, H, H, H] \leqslant H$.

We can thus assume that $H$ has no torsion element outside $\langle x, y\rangle[G, G] G^{p}$. Therefore $\operatorname{dim}\left(H[G, G] G^{p} /[G, G] G^{p}\right)$ is at most 2. Suppose first that the dimension is 2. Then $H$ contains elements of the form $x^{m} y^{r} z^{s} u, y v$, where $m, r, s$ are integers with $p$ not dividing $m$ and $u, v \in[G, G] G^{p}$. From the presentation given in Example 3.2, it follows that

$$
\left[y v, x^{m} y^{r} z^{s} u, x^{m} y^{r} z^{s} u\right]^{p}=[y, x, x]^{p m^{2}}=[y, x, z, x]^{m^{2}} \neq 1
$$

and $H$ again contains $[G, H, H, H]$.

Finally we can assume that $H$ is contained in $\langle t\rangle[G, G] G^{p}$ for some $t \in G$. From the presentation as given in Example 3.2 we see that $G$ has nilpotency class 4 and that $\gamma_{4}(G)$ is cyclic of order $p$. This together with the fact that $G$ is 3-Engel implies $[G, H, H, H] \leqslant\langle[g, t, t, t] \mid g \in G\rangle=1$, the desired result.

The result of the next proposition shows that the class bound for a group in $U_{3}$ with involutions is at least four.

PROPOSITION 3.4. The relatively free 3-Engel group of rank 2 is a $U_{3}$-group.

Proof: Let $G=\langle x, y\rangle$ be the free 3-Engel group of rank 2. Let $H$ be an arbitrary subgroup of $G$. We want to show that $[G, H, H, H] \leqslant H$. First suppose that $H[G, G] G^{2}=$ $G$. Then $H$ contains elements of the form $x u, y v$ with $u, v \in[G, G] G^{2}$. From [4], we know that $G$ has nilpotency class 4 and that $\gamma_{4}(G)=\langle[x, y, y, x]\rangle$ is cyclic of order 2 . But then $[x u, y v, y v, x u]=[x, y, y, x]$, and $H$ therefore contains $\gamma_{4}(G)$. In particular, $[G, H, H, H] \leqslant H$. We can thus assume that $H \leqslant\langle t\rangle[G, G] G^{2}$ for some $t \in G \backslash[G, G] G^{2}$. Using again the fact that $\gamma_{5}(G)=1$ and $\gamma_{4}(G)^{2}=1$, we have $[G, H, H, H] \leqslant\langle[g, t, t, t]|$ $g \in G\rangle=1$.

\section{REFERENCES}

[1] R. Baer, 'Situation der Untergruppen und Struktur der Gruppe', S.B. Heidelberg Akad. Math. Nat. Klasse 2 (1933), 12-17.

[2] R. Dedekind, 'Über Gruppen, deren sämtliche Theiler Normaltheiler sind', Math. Ann. 48 (1897), 548-561. 
[3] D.J. Garrison and L.-C. Kappe, 'Metabelian groups with all cyclic subgroups subnormal of bounded defect', in Proceedings of Infinite Groups 1994 (Walter de Gruyter, 1996), pp. 73-85.

[4] H. Heineken, 'Engelsche Elemente der Länge drei', Ilinois J. Math. 5 (1961), 681-707.

[5] H. Heineken, 'A class of three-Engel groups', J. Algebra 17 (1971), 341-345.

[6] L.-C. Kappe and W.P. Kappe, 'On 3-Engel groups', Bull. Austral. Math. Soc. 7 (1972), 391-405.

[7] S.K. Mahdavianary, 'A special class of three-Engel groups', Arch. Math. 40 (1983), 193-199.

[8] J. E. Roseblade, 'On groups in which every subgroup is subnormal', J. Algebra 2 (1965), 402-412.

[9] D.J.S. Robinson, A course in the theory of groups (Springer-Verlag, Berlin, Heidelberg, New York, 1982).

[10] M. Stadelmann, 'Gruppen, deren Untergruppen subnormal vom Defekt zwei sind', Arch. Math. 30 (1978), 364-371.

[11] G. Traustason, 'On groups in which every subgroup is subnormal of defect at most three', J. Austral. Math. Soc. Ser. A 64 (1998), 1-24.

[12] M. Vaughan-Lee, The restricted burnside problem (Clarendon Press, Oxford, 1993).

SUNY at Binghamton

Binghamton, NY 13902-6000

United States of America

e-mail: menger@math.binghamton.edu
University of Bath

Bath BA2 7AY

England

e-mail: masgt@maths.bath.ac.uk 